\title{
Where postmenopausal hormone therapy stands today
}

\author{
Amos Pines \\ Department of Medicine "T", Ichilov Hospital, Tel-Aviv, The International Menopause Society (IMS), World School \\ for the Study of Menopause (WSSM)
}

\section{INTRODUCTION}

A dramatic change has occurred over the past decade with regard to the use of hormones during the postmenopausal period. In the 90's, the belief in the USA being that there was reason to recommend hormone therapy (HT) for the prevention of coronary artery disease, the guidelines of the American College of Physicians in 1992 were as follows: "All women should consider Hormone Replacement Therapy (HRT). Women with coronary heart disease (CHD) or at risk for CHD are likely to benefit from hormone therapy". ${ }^{1}$ However, things changed in 1998 when the Heart and Estrogen/Progestin Replacement Study (HERS) did not confirm secondary prevention of CHD by HRT, ${ }^{2}$ and four years later the Women's Health Initiative (WHI) data did not support the belief that HRT is effective in primary prevention of $\mathrm{CHD} .^{3}$ The immediate reactions to the WHI results have been expressed in guidelines and recommendations issued by health organizations and medical societies since 2002. For example, the quote taken from the official FDA website, which produced medical up-

Key words: Breast cancer, Coronary artery disease, Hormone replacement therapy, Menopause, Women's health initiative

Address for correspondence:

Amos Pines, MD, Department of Medicine “T”, Ichilov

Hospital, 6 Weizman Street, Tel-Aviv 64239 Israel,

Fax: +972-3-6962715, e-mail: apines@netvision.net.il

Received 28-04-09, Revised 25-06-09, Accepted 20-07-09 dates for consumers of HT, states that: "Hormones should be used at the lowest dose that helps and for the shortest time period needed to achieve treatment goals. Hormones should not be used for prevention of chronic diseases. Hormone treatment is associated with substantial risk for serious adverse events". This was indeed a strong negative message for the public (www.fda.gov/womens/menopause 2002). As a result of the above, positions taken by physicians and women were modified substantially and the number of hormone users has shrunk rapidly. A large survey by the Hormone Foundation conducted in the USA in 2007 yielded some interesting relevant insights into HT. About a quarter of physicians who were asked whether they consider HT as a first line treatment for moderate to severe menopausal symptoms replied that they would prescribe hormones only when alternative methods would not work. Two thirds said that their patients express very much concern as to HT. Furthermore, the majority of patients entertain a high level of misinformation about HT, admitting that the most common sources of information on HT came primarily from friends and relatives, secondarily from the media and the Internet and placing a discussion with their physician as the third option. Although this negative sentiment as regards postmenopausal HT was indeed the result of the early publications from the WHI study in 2002-2003, further data and analyses from the WHI later showed that the actual situation is quite different. Based on current knowledge, which will be detailed in the following sections, it is the duty of the physician to explain differences between clinical facts and fears that are based on misconceptions 
and misinformation with regard to HT.

\section{BENEFITS OF HT ACCORDING TO ACCEPTED INDICATIONS}

No one has ever argued with the basic fact that HT has a positive effect on vasomotor symptoms and vaginal dryness, nor with its potential in reducing the risk for fractures. In fact, based on numerous studies the registration of HT includes the above indications and new products will not be approved if they have failed to demonstrate such benefits according to criteria set by regulatory authorities. In recent years a trend of moving into lower dose hormonal regimens gained popularity, mainly because of the known "rule of thumb" suggesting that smaller doses may be associated with lesser adverse events and risks. There is no need to cite the relevant studies for each and every new low or ultra-low product, but the classical investigations by Notelovitz et al on hot flushes ${ }^{4}$ and Ettinger et al on bone density ${ }^{5}$ clearly demonstrated an interesting dose-response curve for various dosages of estradiol $(0.25 \mathrm{mg}-2 \mathrm{mg})$. However, it should be noted that data on the long-term cardiac or cognitive effects of low dose estrogen or on cancer risk are still lacking. A graded increase in stroke risk was recorded when low dose, standard dose and high dose estrogen regimens were compared in the Nurses' Health Study. ${ }^{6}$

\section{KEY RULES FOR HT}

The International Menopause Society (IMS) published its recommendations on HT, which were based on discussions during two expert meetings, in Budapest (2007) and Zurich (2008). ${ }^{7,8}$ In the Introduction to the document, the IMS acknowledged that there may be geographical variations in the expression of menopause and its treatment, as well as in the incidence of various diseases. The key rules for HT were set as follows: a) HT must be individualized; b) HT should not be recommended without a clear indication for its use; c) there are no reasons to place mandatory limitations on the duration of treatment; d) dosage should be titrated to the lowest effective dose; e) women taking HT should have at least an annual consultation; $f$ ) the risks and benefits of HT differ for women around the time of menopause compared to those for older women. The statement is a key element in understanding the benefit-risk balance of HT, since healthy women using HT in the early postmenopausal period should be reassured that this therapy is safe during the first years of use. One of the major problems with the WHI study was that it examined a relatively old population (mean age 63-64), initiating hormone therapy many years after menopause. In real life, the majority of hormone users start treatment around the beginning of menopause because of vasomotor or urogenital symptoms and take it for a limited period of years. Thus, we should be interested primarily in the benefit-risk balance of HT in the younger age group. Based on recently published analyses from the WHI, we are now able in 2009 to review in detail the data for the age group 50-59 for both treatment arms of the study (estrogen plus progestin $[\mathrm{E}+\mathrm{P}]$ arm and estrogen $[\mathrm{E}]$-alone arm). For this clinical setting, the results are totally different from those presented in 2002. The only remaining significant risk for this age group is venous thrombo-embolism. The updated information is discussed in the next section.

\section{BENEFIT-RISK BALANCE FOR HT WITH A FOCUS ON 50-59 YEAR-OLDS}

\section{Cardiovascular System}

Both arms of the WHI study showed a graded increase in coronary heart disease risk with age and years since menopause at baseline. ${ }^{9}$ However, in the $\mathrm{E}+\mathrm{P}$ arm significant risk was found only for those women in whom hormones were initiated at least 20 years after entering menopause, or after age 70. In the E-alone arm, the same time trend was recorded, but there was no increased risk for coronary events. These results are in line with a recent post-hoc analysis from the Nurses' Health study ${ }^{10}$ and a meta-analysis of randomized controlled trials, ${ }^{11}$ which demonstrated the importance of age in determining the $\mathrm{CV}$ benefit of HT, showing it to be favourable for women below age 60. In fact, several sub-analyses of the WHI E-alone data did show statistical significance in clinical cardiac end points versus placebo for the age group 50-59, ${ }^{12,13}$ the incidence of coronary interventions of all sorts, of myocardial infarctions and of coronary deaths being lower in the E-alone group. Thus, for this young age group estrogen seemed cardioprotective. 
Similar conclusions were drawn by the WHICoronary Artery Calcifications Study. ${ }^{14}$ Computed tomography of the heart was performed after the cessation of the trial in the 50-59 year-old women who participated in the E-alone arm. Coronary calcifications, measured by cardiac tomography, were equivalent to coronary atherosclerotic plaques. Women with various risk factors for CHD had indeed higher calcifications scores, namely, more atherosclerosis, as expected. The only category to show very impressive reduction in calcifications was that of estrogen use, this being even more evident in those women with adherence to therapy of more than $80 \%$ during at least 5 years of therapy. A noteworthy clinical finding from the WHI E+P arm was that the risk for new onset diabetes mellitus was $21 \%$ lower in the hormone users as compared to the placebo arm. ${ }^{15}$ Other major studies pointed to the same effect, the most recent being the Kuopio Osteoporosis Risk Factor and Prevention Study, which recorded a 69\% reduction in the risk for new onset diabetes mellitus. ${ }^{16}$

Estrogen therapy, whether in the form of a contraceptive or as a replacement in the menopause, is associated with a higher risk for stroke. However, because of the very low basal incidence of stroke in the early postmenopausal period, this risk did not reach statistical significance for the age group 50-59 in both arms of the WHI study. ${ }^{17}$ The surplus risk estimate for stroke in hormone users, which was based on the huge cohort of the Nurses' Health Study, amounted to 15 additional cases/100,000 patient-years for the age group 50-54, and 22 additional cases/100,000 patient-years for the age group 55-59. ${ }^{6}$

The case scenario for venous thrombo-embolism is very similar to that for stroke, since the risk is increased in the hormone users. The WHI data pointed to a $30 \%$ increase in the E-alone arm, ${ }^{18}$ and a 2 -fold risk in the $\mathrm{E}+\mathrm{P}$ arm. ${ }^{19}$ These figures, however, should not raise concern since basal risk in the age group 50-59 is in the order of 3-7 extra cases/10,000 patient-years. Moreover, observational studies show that the risk for venous thrombosis with transdermal hormonal preparations is very much lower than that with oral preparations. ${ }^{20}$

\section{BREAST}

The biggest concern with regard to HT is the as- sociation with breast cancer. In 1997, the Collaborative Group on Hormonal Factors in Breast Cancer published an analysis based on 51 epidemiological studies, which showed that the risk for breast cancer is dependent on the duration of therapy. The absolute risk for a 50-year old woman who starts HT and continues to use it long-term was calculated as 2 additional cases $/ 1,000$ women after 5 years of use, and the corresponding numbers for 10 and 15 years of use were 6 and 12 extra cases $/ 1,000,{ }^{21}$ respectively. In the WHI study, a hazard ratio (HR) of 1.24 with borderline significance was recorded for invasive breast cancer in the $\mathrm{E}+\mathrm{P}$ arm during 5.2 years of follow-up. ${ }^{22}$ In contrast with those results, a non-significant reduction in risk (HR 0.8) was demonstrated during 7.1 years in the E-alone arm. ${ }^{23}$ Of note, about a quarter of the WHI participants were exposed to HT before they were recruited to the study. Analyzing the breast cancer data only for women with no prior use of HT showed the same risk as placebo in the $\mathrm{E}+\mathrm{P}$ arm, but a significant breast protection with a HR risk of 0.65 in the E-alone arm.

Two additional issues should be addressed. 1) $\mathrm{HT}$ increases mammographic breast density $(\mathrm{E}+\mathrm{P}$ $>$ E-alone). ${ }^{22,23}$ Increased baseline breast density is a risk factor for breast cancer. However, combining these two facts into a perception which attributes the increased risk for breast cancer in hormone users to the effect on breast density has no clinical basis and there are no data to support such a direct association. 2) Several recent publications reported a concomitant decline in breast cancer incidence and in the number of hormone users in the post-WHI era. These studies claimed that the two trends are linked and therefore prove the concept that cessation of HT is advised since it quickly reduces the risk of breast cancer. ${ }^{24}$ This issue is far too complicated to tackle in the current review since a heated professional debate is still ongoing and no clear-cut conclusions have been drawn (see a press statement by the International Menopause Society (IMS) at http://www.imsociety.org/pages/ comments_and_press_statements/ims_press_statement_04_02_09.html).

\section{CONCLUSIONS}

The WHI study radically altered perceptions on 
the benefit-risk balance of HT by "upgrading" the concerns about breast cancer and cardiovascular outcomes and "downplaying" the benefits associated with quality of life and bone protection, which are the only formal and well supported indications for postmenopausal hormone use. Further analyses from WHI and other major studies, as well as the long debate which was evoked after the first release of WHI data in 2002, may be integrated into the following updated summary that was produced by the IMS: ${ }^{7,8}$

The target population for initiation of HT is usually women up to age 55 .

HT started at the early postmenopausal period in healthy women is safe during the first years of treatment.

An annual consultation with the prescribing physician is recommended.

A decision whether to go on with treatment or stop HT should be made by the individual woman and her healthcare provider, based on symptoms, health status, personal beliefs and expectations. Discussing the individual benefit-risk balance is mandatory.

Transdermal HT and the use of vaginal preparations may reduce certain adverse effects and risks.

Low dose and ultra low dose hormones may be suitable in certain case-scenarios, mainly in older women, presenting a potential lesser risk as compared to standard doses while maintaining efficacy in regard to vaso-motor symptoms and bone protection.

\section{REFERENCES}

1. American College of Physicians, 1992 Guidelines for counseling postmenopausal women about preventive hormone therapy. Ann Intern Med 117: 1038-1042.

2. Hulley S, Grady D, Bush T, et al, 1998 Randomized trial of estrogen plus progestin for secondary prevention of coronary heart disease in postmenopausal women. Heart and Estrogen/progestin Replacement Study (HERS) Research Group. JAMA 280: 605-613.

3. Writing Group for the Women's Health Initiative Investigators, 2002 Risks and Benefits of Estrogen Plus Progestin in Healthy Postmenopausal Women. JAMA 288: 321-333.

4. Notelovitz M, Lenihan JP, McDermott M, et al, 2000 Initial 17 beta-estradiol dose for treating vasomotor symptoms. Obstet Gynecol 95: 726-731.

5. Ettinger B, Genant HK, Steiger P, Madvig P, 1992 Lowdosage micronized 17 beta-estradiol prevents bone loss in postmenopausal women. Am J Obstet Gynecol 166: 479-488.

6. Grodstein F, Manson JE, Stampfer MJ, Rexrode K, 2008 Postmenopausal hormone therapy and stroke: role of time since menopause and age at initiation of hormone therapy. Arch Intern Med 168: 861-866.

7. Pines A, Sturdee DW, Birkhauser M, et al, 2007 IMS updated recommendations on postmenopausal hormone therapy. Climacteric 10: 181-194.

8. Pines A, Sturdee DW, Birkhauser M, et al, 2008 Summary of the first IMS Global Summit on menopause-related issues: HRT in the early postmenopause. Climacteric 11: 267-272.

9. Rossouw JE, Prentice RL, Manson JE, et al, 2007 Postmenopausal hormone therapy and risk of cardiovascular disease by age and years since menopause. JAMA 297: 1465-1477.

10. Grodstein F, Manson JE, Stampfer MJ, 2006 Hormone therapy and coronary heart disease: the role of time since menopause and age at hormone initiation. J Womens Health 15: 35-44.

11. Salpeter SR, Walsh JM, Greyber E, Salpeter EE, 2006 Brief report: Coronary heart disease events associated with hormone therapy in younger and older women. A meta-analysis. J Gen Intern Med 21: 363-366.

12. Hsia J, Langer RD, Manson JE, et al, 2006 Conjugated equine estrogens and coronary heart disease: the Women's Health Initiative. Arch Intern Med 166: 357-365.

13. Saltiki K, Alevizaki M, 2007 Coronary heart disease in postmenopausal women; the role of endogenous estrogens and their receptors. Hormones (Athens) 6: 9-24.

14. Manson JE, Allison MA, Rossouw JE, et al, 2007 Estrogen therapy and coronary-artery calcification. NEJM; 356: 2591-2602.

15. Margolis KL, Bonds DE, Rodabough RJ, et al, 2004 Effect of oestrogen plus progestin on the incidence of diabetes in postmenopausal women: results from the Women's Health Initiative Hormone Trial. Diabetologia 47: 1175-1187.

16. Pentti K, Tuppurainen MT, Honkanen R, et al, 2009 Hormone therapy protects from diabetes: the Kuopio Osteoporosis Risk Factor and Prevention Study. Eur J Endocrinol preprint on-line 25 March 2009.

17. Hendrix SL, Wassertheil-Smoller S, Johnson KC, et al, 2006 Effects of conjugated equine estrogen on stroke in the Women's Health Initiative. Circulation 113: 24252434.

18. Anderson GL, Limacher M, Assaf AR, et al, 2004 Effects of conjugated equine estrogen in postmenopausal women with hysterectomy: the Women's Health Initiative randomized controlled trial. JAMA 291: 1701-1712.

19. Cushman M, Kuller LH, Prentice R, et al, 2004 Estrogen plus progestin and risk of venous thrombosis. JAMA 
292: $1573-1580$.

20. Canonico M, Plu-Bureau G, Lowe GD, Scarabin PY, 2008 Hormone replacement therapy and risk of venous thromboembolism in postmenopausal women: systematic review and meta-analysis. BMJ 336: 1227-1231.

21. Breast cancer and hormone replacement therapy: collaborative reanalysis of data from 51 epidemiological studies of 52,705 women with breast cancer and 108,411 women without breast cancer, 1997 Collaborative Group on Hormonal Factors in Breast Cancer. Lancet 350: 1047-1059.

22. Chlebowski RT, Hendrix SL, Langer RD, et al, 2003
Influence of estrogen plus progestin on breast cancer and mammography in healthy postmenopausal women: the Women's Health Initiative Randomized Trial. JAMA 289: 3243-3253.

23. Stefanick ML, Anderson GL, Margolis KL, et al, 2006 Effects of conjugated equine estrogens on breast cancer and mammography screening in postmenopausal women with hysterectomy. JAMA 295: 1647-1657.

24. Chlebowski RT, Kuller LH, Prentice RL, et al, 2009 Breast cancer after use of estrogen plus progestin in postmenopausal women. N Engl J Med 360: 573-587. 